\title{
Supervivencia de ratas sometidas a diferentes grados de agresión pulmonar
}

\author{
V. CHAMORRO-MARÍNa ${ }^{a}$ M. GARCÍA-DELGADO ${ }^{\text {b }}$ A. RUIZ-AGUILAR ${ }^{\mathrm{b}}$ Y E. FERNÁNDEZ-MONDÉJAR \\ aUnidad Experimental. Hospital Universitario Virgen de las Nieves. Granada. España. \\ bServicio de Cuidados Críticos y Urgencias. Hospital Universitario Virgen de las Nieves. Granada. España.
}

\begin{abstract}
Objetivo. Encontrar un modelo de agresión pulmonar en ratas que se asocie a una mortalidad moderada tras la extubación.

Diseño y ámbito. Estudio experimental realizado en la Unidad Experimental de Investigación del Hospital Universitario Virgen de las Nieves.

Sujetos y método. Se emplearon 45 ratas macho Wistar Kyoto de 250-300 g provistas de agua y comida ad libitum. Las ratas fueron anestesiadas y canuladas a través de un tubo endotraqueal mediante traqueostomía.
\end{abstract}

Intervención. La agresión pulmonar se causaba mediante ventilación mecánica con un volumen corriente de $25 \mathrm{ml} / \mathrm{kg}$ durante 60 min junto con instilación intratraqueal de distintas dosis de solución salina al $0,9 \%$. Las ratas fueron distribuidas al azar en tres grupos de 15 animales cada uno que diferían entre sí por la cantidad de solución salina instilada: grupo I, $0,5 \mathrm{ml}$ solución salina/250 $\mathrm{g}$ de peso de rata; grupo II, $1 \mathrm{ml} / 250 \mathrm{~g}$, y grupo III, $1,5 \mathrm{ml} / 250 \mathrm{~g}$.

Variable de interés principal. La supervivencia después de la extubación se registró cada 5 min durante los primeros $40 \mathrm{~min} y$, después, a las 3 , las 24 y las $72 \mathrm{~h}$ y a los 7 días.

Resultados. Las supervivencias de las ratas que recibieron $0,5,1$ y 1,5 $\mathrm{ml} / 250 \mathrm{~g}$ de solución salina intratraqueal fueron del 60 , el 43 y el $0 \%$ respectivamente. Esta diferencia alcanzó significación estadística entre los grupos que recibieron 0,5 y $1,5 \mathrm{ml} / 250 \mathrm{~g}(\mathrm{p}=0,003)$.

Financiación parcial por la Fundación del Hospital U. Virgen de las Nieves (FIBAO) y FIS 06/1097.

Correspondencia: Dra. V. Chamorro.

Unidad de Investigación Experimental. Servicio de Cuidados Críticos

y Urgencias. Hospital Universitario Virgen de las Nieves.

Dr. Azpitarte, 4. 18012 Granada. España.

Correo electrónico:vchamo26@hotmail.com

Manuscrito aceptado el 30-10-2008.
Conclusiones. La supervivencia de las ratas sometidas a ventilación mecánica con un volumen corriente moderadamente alto está influida por dosis crecientes de solución salina intratraqueal, lo que puede ser de interés para el diseño de estudios que analicen el efecto de intervenciones sobre la mortalidad.

PALABRAS CLAVE: Ventilación mecánica. Supervivencia. Agresión pulmonar.

\section{SURVIVAL OF RATS SUBJECTED TO DIFFERENT LEVELS OF PULMONARY INJURY}

Objective. To determine a pulmonary injury model in rats that is associated with moderate mortality after extubation.

Design and setting. An experimental study in an animal model of ventilator-induced lung injury in the animal research laboratory in Virgen de las Nieves University Hospital.

Subjects and method. A total of $\mathbf{4 5}$ male WistarKyoto rats weighing $250-300 \mathrm{~g}$ received food and water ad libitum. The rats were anesthetized and a tracheotomy was performed by insertion of endotracheal tube by tracheotomy.

Interventions. Pulmonary injury due to mechanical ventilation was maintained for $60 \mathrm{~min}$ with high tidal volume $(25 \mathrm{ml} / \mathrm{kg})$ combined with intratracheal instillation of different doses of $0,9 \%$ saline solution. Rats were randomly distributed into 3 groups (15 animals in each group) with different amounts of instilled saline solution: group I, 0.5 $\mathrm{ml} / 250 \mathrm{~g}$ body weight; group II, $1 \mathrm{ml} / 250 \mathrm{~g}$ body weight, and group III, $1.5 \mathrm{ml} / 250 \mathrm{~g}$ body weight.

Main measurements. Survival of animals after extubation was recorded every 5 min for the first $40 \mathrm{~min}$ and then at $3 \mathrm{~h}, 24 \mathrm{~h}, 72 \mathrm{~h}$, and 7 days.

Results. Survival in rats that received $0.5,1$ and $1.5 \mathrm{ml} / 250 \mathrm{~g}$ of intratracheal saline solution was 
$60 \%, 43 \%$ and $0 \%$ respectively, with statistically significant differences between groups receiving 0.5 and $1.5 \mathrm{ml} / 250 \mathrm{~g}(\mathrm{p}=0.003)$.

Conclusions. Survival in rats mechanically ventilated with high moderate volume is influenced by increased doses of intratracheal saline solution and this is important to design studies that analyze the effect the interventions on mortality.

KEY WORDS: Mechanical ventilation. Survival. Pulmonary aggression.

\section{INTRODUCCIÓN}

Generalmente, los estudios experimentales con animales se centran en el análisis de parámetros fisiológicos o histológicos, pero son escasos los estudios que analizan el efecto de una determinada acción sobre la mortalidad. Aunque el análisis de parámetros fisiológicos puede orientar sobre el beneficio o el perjuicio de una determinada acción, no cabe duda de que la mortalidad supone un avance a la hora de interpretar estos efectos.

La ventilación mecánica (VM) con volumen corriente (VC) moderadamente alto durante periodos cortos puede causar lesión pulmonar y edema pulmonar en ratas por sobredistensión del pulmón (volutrauma $)^{1,2}$. Este tipo de agresión puede producir alteraciones tanto fisiológicas como morfológicas similares al daño alveolar difuso observado en el síndrome del distrés respiratorio agudo (SDRA) ${ }^{1-8}$. Estos efectos se han demostrado generalmente empleando un volumen corriente extremadamente alto, entre 45 y $90 \mathrm{ml} / \mathrm{kg}^{9}$, lo que ciertamente provoca el cuadro de daño alveolar difuso, pero éste es de una gravedad tal que lo hace irreversible y sin posibilidad de supervivencia ante cualquier medida terapéutica.

Nuestro objetivo ha sido encontrar un modelo de lesión pulmonar experimental que comporte una mortalidad moderada, de forma que permita en posteriores estudios valorar la eficacia de planes terapéuticos específicos. Dado que algunas de las actuaciones terapéuticas consisten en administrar intratraquealmente medicación o sustancias diluidas en solución salina y que -particularmente en animales pequeños- pueden comportarse como «edema» e interferir en el pronóstico, hemos diseñado un modelo que combina la sobredistensión alveolar moderada con la provocación de edema alveolar por inundación con solución salina.

\section{MÉTODO}

\section{Animales de experimentación}

Se emplearon 45 ratas macho Wistar-Kyoto de 250-300 g provistas de agua y comida ad libitum mantenidas con un ciclo de 12 h de día y 12 h de noche. Los animales fueron adquiridos en la Universidad de Granada. El estudio fue aprobado por el Comité Ético y de Investigación del Hospital Universitario Virgen de las Nieves de Granada. En todo momento se han respetado las leyes de manejo y experimentación animal vigentes en nuestro país (Real Decreto 1201/2005).

\section{Anestesia}

Como premedicación se utilizó atropina $0,05 \mathrm{mg} /$ $\mathrm{kg}$ (c.s.) y acepromacina $2 \mathrm{mg} / \mathrm{kg}$ (i.m.), clorpromacina $10 \mathrm{mg} / \mathrm{kg}$ (i.m.). Tras la premedicación, se procedía a la anestesia de los animales con un coctel de $5 \mathrm{ml}$ de ketamina $(50 \mathrm{mg} / \mathrm{ml}), 2 \mathrm{ml}$ de diazepam (10 $\mathrm{mg} / 2 \mathrm{ml}), 1 \mathrm{ml}$ de atropina $(1 \mathrm{mg} / \mathrm{ml})$.

De esta mezcla se administraron por vía intraperitoneal $0,8 \mathrm{ml} / 300 \mathrm{~g}$ en machos. Pasados $10 \mathrm{~min}$ de la inyección, la rata estaba perfectamente anestesiada. Con la dosis de anestesia descrita, la mayoría de los animales permanecen durante 60 min suficientemente analgesiados, pero despiertos, para que puedan respirar espontáneamente. En el caso de que se detectara algún signo de malestar, como movimiento de cabeza o intento de motilidad, se administraba $0,2 \mathrm{ml}$ de ketamina sin diazepam (las veces que fuera necesario).

\section{Ventilación mecánica}

Tras la sedación, los animales eran colocados en una placa caliente para poder mantener la temperatura corporal del animal y, a continuación, se les hacía una traqueostomía y se les insertaba un catéter de $2 \mathrm{~mm}$ (B/Braun, São Gonçalo, Rio de Janeiro, Brasil). Los animales eran ventilados con un respirador (modelo 683; Harvard Apparatus, South Natick, Massachusetts, Estados Unidos) conectado a una bomba que suministra oxígeno, resultando una $\mathrm{FiO}_{2}$ de $0,5-0,6$, con un volumen corriente (VC) de $25 \mathrm{ml} / \mathrm{kg}$, frecuencia respiratoria de $40 / \mathrm{min}$ y presión positiva espiratoria final (PEEP) de $4 \mathrm{cmH}_{2} \mathrm{O}$. Se registró la presión en las vías aéreas (presión máxima) al inicio de la ventilación mecánica (VM), tras la instilación de solución salina y al final del experimento mediante un transductor de presión (Transpac, Abbot, Chicago, Illinois, Estados Unidos) conectado a un monitor (Hellige Servomed, Alemania).

\section{Lesión pulmonar}

Tras la conexión a VM, se procedía a instilar en la tráquea distintas dosis de solución salina, según el grupo al que correspondiera $(0,5,1$ y $1,5 \mathrm{ml} / 250 \mathrm{~g})$.

\section{Grupos}

Grupo I, ventilación con VC de $25 \mathrm{ml} / \mathrm{kg}$ durante 60 $\min +0,5 \mathrm{ml} / 250 \mathrm{~g}$ de solución salina intratraqueal; grupo II, ventilación con VC de $25 \mathrm{ml} / \mathrm{kg}$ durante 60 $\mathrm{min}+1 \mathrm{ml} / 250 \mathrm{~g}$ de solución salina intratraqueal, y grupo III, ventilación con VC de $25 \mathrm{ml} / \mathrm{kg}$ durante 60 $\mathrm{min}+1,5 \mathrm{ml} / 250 \mathrm{~g}$ de solución salina intratraqueal.

\section{Desconexión de la ventilación mecánica}

Concluidos los 60 min de VM, los animales eran desconectados del respirador y se permitía la respiración espontánea a través de la traqueostomía en una atmósfera rica en $\mathrm{O}_{2}$ durante 15 min. A medida que los 
animales iban recobrando motilidad (15 min después), se cerraba el traqueostoma con una seda y se trasladaban a una jaula individual con libre acceso a la comida y el agua y una higiene adecuada, registrándose su evolución y, en su caso, el momento de fallecimiento.

\section{Análisis de supervivencia}

Tras la desconexión del respirador, se monitorizaba la supervivencia cada $5 \mathrm{~min}$ hasta llegar a los $40 \mathrm{~min}$, y después a las 3 , las 24 y las 72 h y a los 7 días.

\section{Análisis de datos}

Los datos se expresan como media \pm error estándar. El programa SPSS 13.0 se utiliza para el análisis estadístico. Las medias de la variables numéricas entre los grupos I, II y III se comparan usando un ANOVA. El test de Tukey se aplica cuando las varianzas de las variables son iguales y el test de Dunnet cuando son diferentes. Cuando se comparan dos medias, se aplica la prueba de la $t$ de Student. Para el análisis de supervivencia utilizamos curvas de Kaplan-Meier. Para la comparación de las curvas de supervivencia, el estadístico de contraste que utilizamos es el log-rank test, que indica la $\mathrm{p}$ general y si hay diferencias entre grupos.

\section{RESULTADOS}

Se observa una ligera tendencia a disminuir en los valores de presión en vías aéreas en los grupo I y III, y diferencias estadísticamente significativas entre el inicio y el final de la VM ( $\mathrm{p}=0,003)$ (tabla 1).

La supervivencia de las ratas ventiladas con un VC moderadamente alto fue superior entre las sometidas a solución salina intratraqueal $0,5 \mathrm{ml} / 250 \mathrm{~g}$ con respecto a las que recibieron $1,5 \mathrm{ml} / 250 \mathrm{~g}(\mathrm{p}=0,003)$. La supervivencia de las ratas que recibieron $1 \mathrm{ml} / 250$ $\mathrm{g}$ fue del $43 \%$, aunque no se alcanzaron diferencias estadísticamente significativas con los otros grupos $(\mathrm{p}=0,394$ frente al grupo que recibió $0,5 \mathrm{ml} \mathrm{y} \mathrm{p}=$ 0,054 frente al grupo que recibió $1,5 \mathrm{ml}$ ).

Quince minutos después de la extubación, murieron 5 de 15 animales del grupo I, 6 del grupo II y 7 del grupo III. Entre los minutos 15 y 40 las diferencias se hacen evidentes, sobre todo en el grupo al que se administró 1,5 ml/250 g, en el que sólo sobrevivió

TABLA 1. Medición de presiones en las vías aéreas al principio y al final de la ventilación mecánica

\begin{tabular}{|l|c|c|}
\hline & PVA $_{0}$ & ${ }^{2 V A}$ \\
\hline Grupo I & $27,93 \pm 0,46$ & $26,87 \pm 0,48$ \\
Grupo II & $26,86 \pm 0,35$ & $27 \pm 0,43$ \\
Grupo III & $27,29 \pm 0,31$ & $25,93 \pm 0,24 *$ \\
\hline
\end{tabular}

$\mathrm{PVA}_{0}$ : presión en vías aéreas al inicio de la ventilación mecánica; $\mathrm{PVA}_{1}$ : presión en vías aéreas al final de la ventilación mecánica.

*Diferencias estadísticamente significativas en el grupo III al principio y al final del experimento $(\mathrm{p}=0,003)$.

Los valores expresan medias \pm error estándar.
1 rata. A los 7 días, la supervivencia se mantenía en los grupos a los que se administró solución salina intratraqueal a 0,5 y $1 \mathrm{ml} / 250 \mathrm{~g}$ (fig. 1).

\section{DISCUSIÓN}

Este estudio demuestra que, en ratas ventiladas con un $\mathrm{VC}$ moderadamente alto, la administración de dosis crecientes de solución salina intratraqueal se acompaña de un progresivo incremento en la mortalidad. La instilación de solución salina intratraqueal en dosis altas (1,5 $\mathrm{ml} / 250 \mathrm{~g}$ ) se acompaña de una total mortalidad; en cambio, cuando la cantidad de solución salina intratraqueal es menor $(0,5$ y $1 \mathrm{ml} / 250 \mathrm{~g}$ ) permite obtener supervivencias del 60 y el $43 \%$ respectivamente, lo que hace muy útiles a estos modelos experimentales para estudios de intervención terapéutica sobre estos animales.

La mortalidad durante los primeros $15 \mathrm{~min}$ tras la extubación es muy similar en los tres grupos. Esto puede deberse a que durante ese periodo los animales se encontraban respirando una atmósfera rica en oxígeno que suministra el apoyo suficiente para poder mantenerse vivos. Sin embargo, cuando se retira el aporte de oxígeno, se aprecia un descenso progresivo de la supervivencia, en particular en el grupo que recibió $1,5 \mathrm{ml} / 250 \mathrm{~g}$, lo que indica que la lesión pulmonar era tan grave que estas ratas se mantenían vivas por el aporte de oxígeno suministrado.

El leve descenso de la presión en vías aéreas observado en el grupo III es difícil de interpretar; por una parte, el significado biológico de un descenso de poco más de 1 $\mathrm{cmH}_{2} \mathrm{O}$ puede ser discutible, aunque podría estar en relación con una cierta flacidez tisular como consecuencia de mayor estrés tisular a los 60 min del experimento ${ }^{10}$.

Pocos estudios han analizado la supervivencia de ratas sometidas a sobredistensión alveolar. Nin et al ${ }^{11}$ encuentran una supervivencia del $50 \%$ de las ratas sometidas a VM con un VC de $35 \mathrm{ml} / \mathrm{kg}$. Nosotros encontramos una supervivencia del $60 \%$ en el grupo que recibió $0,5 \mathrm{ml} / 250 \mathrm{~g}$, pero nuestros animales fueren ventilados con un VC claramente inferior $(25 \mathrm{ml} /$ $\mathrm{kg}$ ), lo que sin duda ha influido en la mayor tasa de supervivencia en nuestro estudio.

\section{Limitaciones}

Este estudio ha sido realizado en animales pequeños (ratas), y es conocido que la VM con alto VC tiene efectos mucho más intensos en este tipo de animales que en otros de mayor tamaño ${ }^{12-16}$. Nuestro grupo ha demostrado que en animales de mayor tamaño (cerdos) la $\mathrm{VM}$ con $50 \mathrm{ml} / \mathrm{kg}$ de $\mathrm{VC}$ apenas origina lesión pulmonar ${ }^{17}$, por lo que evidentemente nuestros resultados sólo serán aplicables a la especie con la que ha sido desarrollado.

Además, algunos autores consideran que el modelo de lesión pulmonar por alto $\mathrm{VC}$ no tiene traducción clínica aplicable. Nuestra opinión en este aspecto es diferente: nosotros creemos que hay circunstancias clínicas que lo hacen muy verosímil, como casos de intubación selectiva de un bronquio principal que puede pasar inadvertida durante ciertos lapsos. Este 


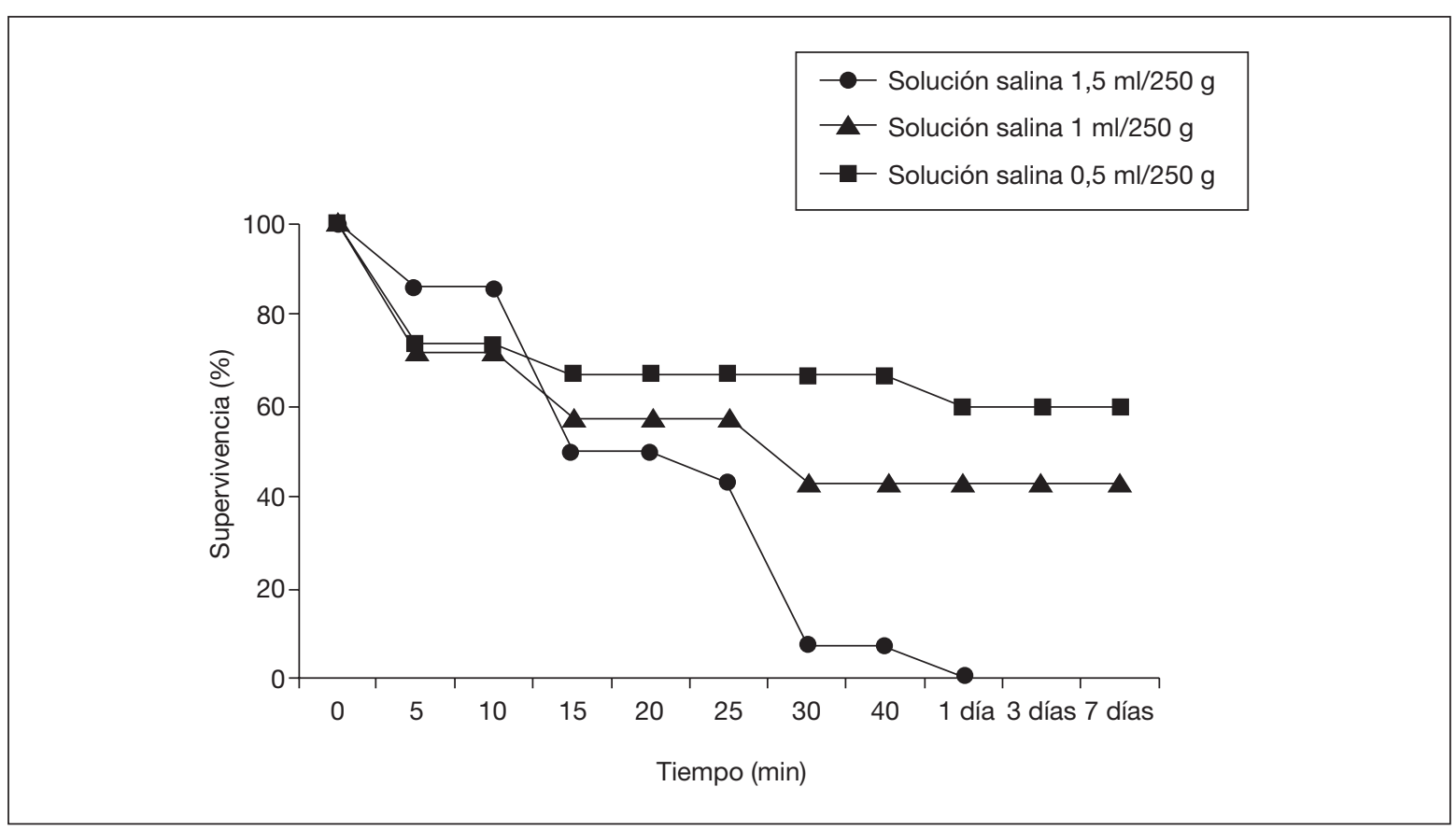

Figura 1. Perfil de supervivencia de los animales según la dosis de solución salina intratraqueal suministrada. Los valores se expresan como proporciones. *Diferencias significativas entre los grupos I y III $(p=0,003)$

es un hecho no excepcional que puede reproducir las circunstancias de nuestro estudio.

\section{CONCLUSIONES}

La supervivencia en ratas sometidas a VM con un VC moderadamente alto está influida por dosis crecientes de solución salina intratraqueal, lo que puede ser de interés para el diseño de estudios que analicen el efecto de intervenciones sobre la mortalidad.

\section{AGRADECIMIENTOS}

Los autores agradecen a Sabina Pérez Vicente por la ayuda prestada en el análisis estadístico.

\section{BIBLIOGRAFÍA}

1. Dreyfuss D, Basset G, Soler P, Saumon G. Intermittent positivepressure hyperventilation with high inflation pressures produces pulmonary microvascular injury in rats. Am Rev Respir Dis. 1985;132:880-4.

2. Gordo Vidal F, Delgado Arnaiz C, Calvo Herranz E. Lesión pulmonar inducida por la ventilación mecánica. Med Intensiva. 2007;31:18-26.

3. Corbridge TC, Wood LDH, Crawford GP, Chudoba MJ, Yanos J, Sznajder JI. Adverse effects of large tidal volume and low PEEP in canine acid aspiration. Am Rev Respir Dis. 1990;42:311-5.

4. Dreyfuss D, Soler P, Basset G, Saumon G. High inflation pressure pulmonary edema. Respective effects of high airway pressure, high tidal volume, and positive end-expiratory pressure. Am Rev Respir Dis. 1988;137:1159-64.

5. Dreyfuss D, Soler P, Saumon G. Spontaneous resolution of pulmonary edema caused by short periods of cyclic overinflation. J Appl Physiol. 1992;72:2081-9.

6. Sznajder JI, Ridge KM, Saumon G, Dreyfuss D. Lung injury induced by mechanical ventilation. En: Matthay M, Ingbar D, edi- tores. Pulmonary Edema. New York: Marcel Dekker; 1998. p. 413-30.

7. Tremblay L, Valenza F, Ribeiro SP, Li J, Slutsky AS. Injurious ventilatory strategies increase cytokines and c-fos mRNA expression in an isolated rat lung model. J Clin Invest. 1997;99:944-52.

8. Webb HH, Tierney DF. Experimental pulmonary edema due to intermittent positive pressure ventilation with high inflation pressures: protection by positive end-expiratory pressure. Am Rev Respir Dis. 1974;110:556-65.

9. Dreyfuss D, Soler P, Saumon G. Mechanical ventilation-induced pulmonary edema. Interaction with previous lung alterations. Am J Respir Crit Care Med. 1995;151:1568-75.

10. Ventrice EA, Martí-Sistac O, Gonzalvo R, Villagrá A, LópezAguilar J, Blanch L. Mecanismos biofísicos, celulares y modulación de la lesión pulmonar inducida por la ventilación mecánica. Med Intensiva. 2007;31:73-82.

11. Nin $N$, Penuelas $O$, De Paula $M$. Rats surviving after high tidal volume ventilation show marked reversible pulmonary and systemic inflammation and long-term survival. Intensive Care Med. 2008;34:948-56.

12. Berthiaume Y, Staub NC, Matthay MA. Beta-adrenergic agonists increase lung liquid clearance in anesthetized sheep. J Clin Invest. 1987;79:335-43.

13. Berthiaume Y, Broaddus VC, Gropper MA, Tanita T, Matthay MA. Alveolar liquid and protein clearance from normal dog lungs. J Appl Physiol. 1988;65:585-93.

14. Matthay MA, Berthiaume Y, Staub NC. Long-term clearance of liquid and protein from the lungs of unanesthetized sheep. J Appl Physiol. 1985;59:928-34.

15. Matthay MA, Landolt CC, Staub NC. Differential liquid and protein clearance from the alveoli of anesthetized sheep. J Appl Physiol. 1982;53:96-104.

16. Smedira N, Gates L, Hastings R, Jayr C, Sakuma T, Pittet JF, et al. Alveolar and lung liquid clearance in anesthetized rabbits. J Appl Physiol. 1991;70:1827-35.

17. García-Delgado M, Navarrete-Sánchez I, Colmenero M, Touma-Fernández A, López-Cuervo JE, Hassan-Montero L, et al. Intermittent alveolar overdistension for 30 or 240 minutes does not produce acute lung injury in normal pig lung. J Surg Res. 2006;131:233-40. 